\title{
Modelling traumatic brain injury in pedestrian involved in backover collisions
}

\author{
Atsutaka Tamura* \\ Department of Mechanical and Aerospace Engineering, \\ Graduate School of Engineering, \\ Tottori University, \\ Tottori, Tottori 680-8552, Japan \\ Email: a-tamura@tottori-u.ac.jp \\ *Corresponding author
}

\section{King $\mathrm{H}$. Yang}

Wayne State University, Detroit, Michigan 48201, USA

Email: king.yang@wayne.edu

\begin{abstract}
Backover collisions causing Traumatic Brain Injuries (TBIs) are underreported, and its severity may have been overlooked and underestimated. We conducted a series of pedestrian impact simulations involving backover collisions with a reversing vehicle, at a low speed of $10 \mathrm{~km} / \mathrm{h}$, to determine the risk of sustaining severe TBIs. Our modelling studies revealed a significant risk despite the 'moderate' impact configuration applied. By systematically performing injury analyses based on selected mechanical parameters, we found that TBI risk involved in primary head strike with a striking vehicle was almost negligible because of the low-speed collision, but significant injuries result from ground impact. Our study also demonstrated that pedestrians are potentially at a greater risk for TBI when struck by a Sport Utility Vehicle (SUV) than a conventional sedan, because the impact energy would be effectively transmitted from the SUV via its flat rear surface with a steep angle.

Keywords: TBI; traumatic brain injury; pedestrian; backover collision; reversing vehicle; ground impact.

Reference to this paper should be made as follows: Tamura, A. and Yang, K.H. (2020) 'Modelling traumatic brain injury in pedestrian involved in backover collisions', Int. J. Vehicle Safety, Vol. 11, No. 3, pp.229-246.
\end{abstract}

\section{Introduction}

Emphases on pedestrian safety have been placed on a relatively severe "frontal" collision rather than a low-speed impact with a reversing vehicle, i.e., backover crash. According to the National Highway Traffic Safety Administration (NHTSA), backover crash can be described as "a specifically-defined type of incident, in which a non-occupant of a vehicle (i.e., a pedestrian or cyclist) is struck by a vehicle moving in reverse" (NHTSA, 2010, 2019). On the basis of the NHTSA Not-in-Traffic Surveillance system, Austin 
(2008) estimated that the annual backover injuries in the USA totalled approximately 18,000 , in which 4000 incidents were on-road accidents reported by the police, whereas the remaining 14,000 injuries were related to off-road accidents. This statistics indicates that official road injury databases may record only crashes on public roads, excluding a significant portion of injuries, which occur off public traffic routes, including private driveways and parking lots (Keall et al., 2017).

Recently, Fildes et al. (2017) conducted an analysis on national police-reported backover collision data from four countries, i.e., the USA, Germany, the UK and Australia, and showed that the annual average percentage of pedestrians involved in backover collisions was approximately $7.4 \%$ compared with all pedestrian injuries. Further, the proportion of backover injuries to all pedestrian collisions was found to be relatively similar among the four Western countries stated above and concluded that it potentially reflects fewer reported incidences and/or less severe crashes. However, the data revealed that these backover incidents sometimes resulted in fatal outcomes for the involved pedestrians and more than $90 \%$ of the victims involved a severe (fatal or non-fatal) injury, implying that the severity of backover injuries are substantially underestimated at present.

Despite the limited coverage of off-road accidents, the importance of backover injuries has been identified as considerable and unignorable (Lindman et al., 2011; Decker et al., 2016), even though the impact velocities are generally low $(10 \mathrm{~km} / \mathrm{h}$ or below) in such reversing accidents. They further highlighted that AIS 2+ (Abbreviated Injury Scale $\geq 2$, greater than or equal to moderate level) injuries to the head primarily occurred secondary to impact with the ground and accounted for $12.3-27.3 \%$ of the total, in which concussions followed by haemorrhage and fractures were the most common type of injuries.

Our previous studies (Tamura et al., 2016, 2018; Tamura and Yang, 2019) revealed that pedestrians are potentially exposed to greater risk of sustaining a severe Traumatic Brain Injury (TBI) involving "frontal" collisions (wrap-around and fender vault impacts) and sideswipe accidents, even under low-speed impact velocities at $20-25 \mathrm{~km} / \mathrm{h}$ when the ground impact event is considered. Nevertheless, pedestrian impact simulations with a reversing vehicle have not been addressed so far. Hence, in the present study, a series of backover crashes were conducted, and we investigated the risk of sustaining TBI based on the selected mechanical parameters. The objective of this study was to understand typical responses of a struck pedestrian involved in backover crashes and to determine whether the risk of sustaining life-threatening TBIs is likely low or high in this reverse impact setting.

\section{Methods}

\subsection{Simulation setup}

Full-scale vehicle Finite Element (FE) models are available at the archive of Crash Simulation Vehicle Models (NHTSA, Washington, DC, USA). In the present study, a Sport Utility Vehicle (SUV), Ford Explorer, and a sedan, Ford Taurus, were selected as striking vehicles. An American adult male 50th percentile pedestrian model (THUMS ver. 3, Toyota Central R\&D Labs., Inc., Nagakute, Aichi, Japan) was employed as a struck pedestrian FE model, which replicated a gaiting posture with a left leg placed 
forward. This pedestrian model was not fully validated against ground impact events due to the paucity of available experimental data. However, it was confirmed previously (Tamura et al., 2016) that the model-predicted throw distance for the impacted pedestrian was highly comparable with that reported in real-world accident data (Simms et al., 2004; Wood et al., 2005). Geometric dimensions of the rear surface configuration of the striking vehicles are summarised in Figure 1 and Table 1. The numbers of elements and parts comprising the human body model were 203,238 and 1414, respectively. Also, the corresponding numbers for the SUV were 619,751 and 756, and for the sedan were 982,389 and 780, respectively. Striking vehicles were lowered by $65 \mathrm{~mm}$ to the ground so that tyre compression due to vehicle weight would be considered, and a series of lowspeed backover crashes were reconstructed with a reversing vehicle at an impact speed of $10 \mathrm{~km} / \mathrm{h}(-2.8 \mathrm{~m} / \mathrm{s})$ with a deceleration pulse of $0.5 \mathrm{~g}$ 's. Additionally, gravitational force was given to the pedestrian model at $t=0$, while any preload due to its body weight was not considered in this study.

Figure 1 Geometric dimension of the rear surface configuration of striking vehicles (left: SUV; right: sedan)
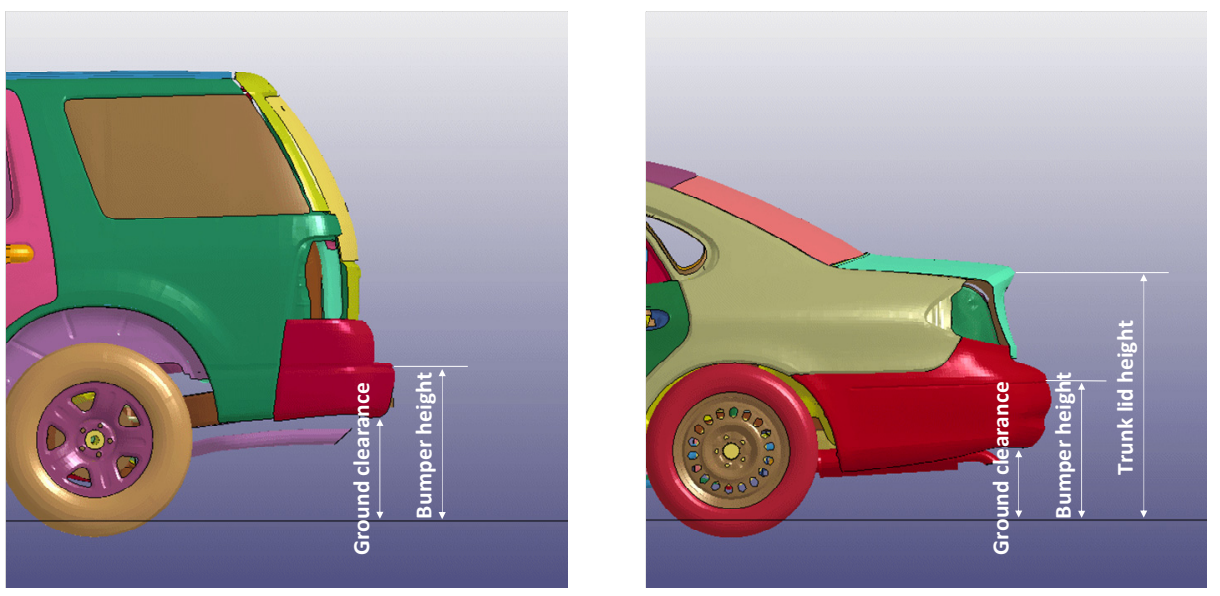

Table 1 Geometric dimension of the rear surface configuration of striking vehicles simulated in this study

\begin{tabular}{ccccc}
\hline $\begin{array}{c}\text { Vehicle } \\
\text { type }\end{array}$ & $\begin{array}{c}\text { Trunk lid height } \\
(\mathrm{mm})\end{array}$ & $\begin{array}{c}\text { Rear bumper } \\
\text { height }(\mathrm{mm})\end{array}$ & $\begin{array}{c}\text { Ground clearance } \\
(\mathrm{mm})\end{array}$ & $\begin{array}{c}\text { Rear window } \\
\text { angle }(\text { deg })\end{array}$ \\
\hline SUV & N/A & 575 & 400 & 65 \\
Sedan & 930 & 525 & 290 & 25 \\
\hline
\end{tabular}

Note: Trunk lid height was not available for an SUV.

Although specific information on pedestrian movements and positions prior to an impending collision is usually lacking for the backover accident type (Lindman et al., 2011), the vast majority of accidents occur at the rear-end of the reversing vehicles (Decker et al., 2016). Thus, initial impact configurations were designed such that a standing or moving pedestrian would be hit laterally by the corner of rear left, rear right or the end of rear centre, while the pedestrian was transversely travelling in the perpendicular direction to the moving vehicle at $V_{y}=0.0$ (standing), 1.3 (walking), 2.7 
(jogging), or 4.0 (running) $\mathrm{m} / \mathrm{s}$, respectively (see Figure 2 and Table 2). When a pedestrian was hit at the left or right corners, it was positioned at $700 \mathrm{~mm}$ away from the vehicle centre line. Of note, the positive $X$-, $Y$ - and $Z$-axes indicate backward, left-to-right and inferior-to-superior directions, respectively, and the throw distance of a struck pedestrian was measured on the basis of the moving distance of the head in an $X-Y$ plane. Of another note is that the given deceleration pulse gradually decreased from $0.5 \mathrm{~g}$ to a different asymptotic value depending on each vehicle weight (see Figure 3).

Figure 2 Typical initial impact configurations with reversing vehicles (left: SUV vs. pedestrian positioned at centre; right: sedan vs. pedestrian positioned at right corner). An impact velocity was set at $10 \mathrm{~km} / \mathrm{h}$ with a deceleration pulse of $0.5 \mathrm{~g}$ 's. (right and left positions were $700 \mathrm{~mm}$ away from the centreline)
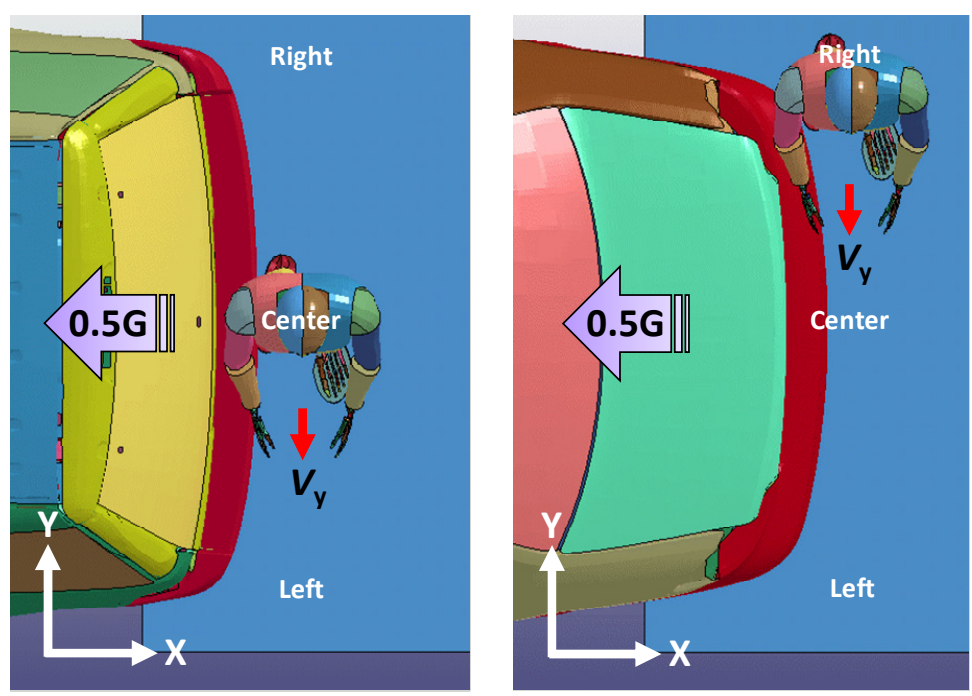

Table 2 Allocation of selected design parameters determining various impact configurations for a series of backover crash simulations $(N=24)$

\begin{tabular}{cccc}
\hline Case ID & Vehicle type & Pedestrian position & Pedestrian speed, $V_{y}(\mathrm{~m} / \mathrm{s})$ \\
\hline suv 01 & SUV & Right & 0.0 \\
suv 02 & SUV & Right & 1.3 \\
suv 03 & SUV & Right & 2.7 \\
suv 04 & SUV & Right & 4.0 \\
suv 05 & SUV & Centre & 0.0 \\
suv 06 & SUV & Centre & 1.3 \\
suv 07 & SUV & Centre & 2.7 \\
suv 08 & SUV & Centre & 4.0 \\
suv 09 & SUV & Left & 0.0 \\
suv 10 & SUV & Left & 1.3 \\
suv 11 & SUV & Left & 2.7 \\
suv 12 & SUV & Left & 4.0 \\
\hline
\end{tabular}


Table 2 Allocation of selected design parameters determining various impact configurations for a series of backover crash simulations $(N=24)$ (continued)

\begin{tabular}{cccc}
\hline Case ID & Vehicle type & Pedestrian position & Pedestrian speed, $V_{y}(\mathrm{~m} / \mathrm{s})$ \\
\hline sdn 01 & Sedan & Right & 0.0 \\
sdn 02 & Sedan & Right & 1.3 \\
$\operatorname{sdn} 03$ & Sedan & Right & 2.7 \\
$\operatorname{sdn} 04$ & Sedan & Right & 4.0 \\
$\operatorname{sdn} 05$ & Sedan & Centre & 0.0 \\
$\operatorname{sdn} 06$ & Sedan & Centre & 1.3 \\
$\operatorname{sdn} 07$ & Sedan & Centre & 2.7 \\
$\operatorname{sdn} 08$ & Sedan & Centre & 4.0 \\
$\operatorname{sdn} 09$ & Sedan & Left & 0.0 \\
$\operatorname{sdn} 10$ & Sedan & Left & 1.3 \\
$\operatorname{sdn} 11$ & Sedan & Left & 2.7 \\
$\operatorname{sdn} 12$ & Sedan & Left & 4.0 \\
\hline
\end{tabular}

Figure 3 Typical changes in a deceleration pulse of striking vehicles. (a) SUV (suv 03) (b) Sedan (sdn 07)

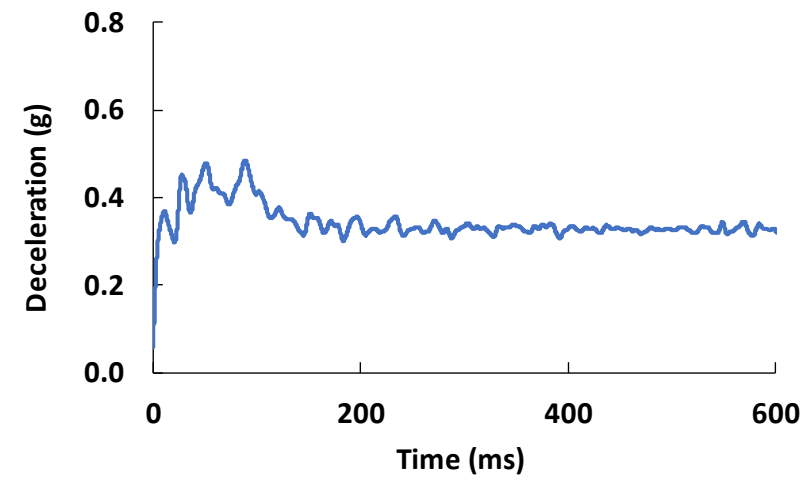

(a)

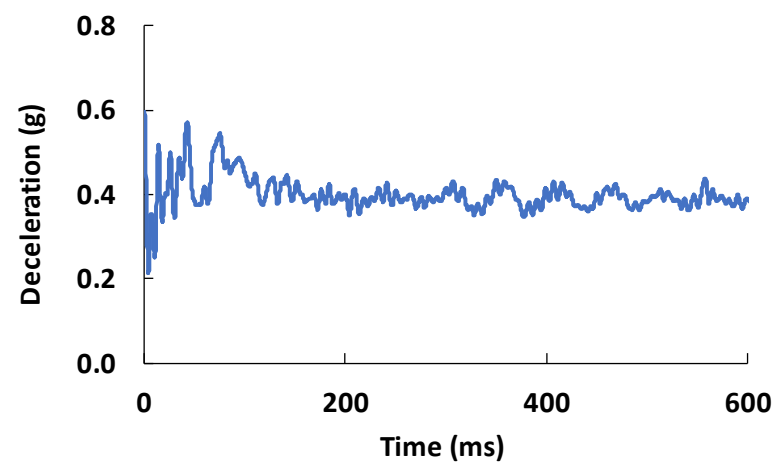

(b) 
As for the pedestrian-ground interaction, a well-validated contact model is yet to be available (Crocetta et al., 2015). In this study, therefore, the ground was modelled as a rigid plane with a friction coefficient of 0.6 assuming a dry-asphalt road surface (Tamura et al., 2016). Note that the friction coefficient, 0.3 , was used throughout the current work for replicating the mechanical interaction of a vehicle-pedestrian contact in accordance with a couple of previous studies (Simms and Wood, 2006; Crocetta et al., 2015; Tamura et al., 2016). In total, 24 impact simulations were performed. We used LS-DYNA v971 R6.1.1 (Livermore Software Technology Corp., Livermore, CA, USA) for the entire numerical analyses throughout the study.

\subsection{Injury assessment parameters}

Since the current study was conducted in a low-speed impact severity, the primary head strike against a striking vehicle was mostly eliminated and its effect was negligible, allowing us to focus on secondary head strike involved in ground impact event. We chose four mechanical parameters to assess the risk of sustaining TBI in reference to our previous studies (Tamura et al., 2016, 2018; Tamura and Yang, 2019), i.e., Head Injury Criterion $\left(\mathrm{HIC}_{15}\right)$, maximum head rotational acceleration $\left(\ddot{\theta}_{\max }\right)$, Cumulative Strain

Damage Measure (CSDM), and Brain Trauma Index (BTI). Specifically, $\mathrm{HIC}_{15}$ was computed on the basis of a resultant translational acceleration pulse $a(t)$ applied to the Centre of Gravity (CG) of the head as given by equation (1), which is widely used in the automotive safety area with the time window of $t_{2}-t_{1}=15 \mathrm{~ms}$ or less. Similarly, $\ddot{\theta}_{\max }$ was computed on the basis of a resultant rotational acceleration pulse $\ddot{\theta}(t)$ applied to the head CG as given by equation (2) with the fixed time clip of $t_{4}-t_{3}=3 \mathrm{~ms}$ (Tamura et al., 2016). In the present study, the 3-ms clip was selected to surely capture the peak values involved in ground impact events, avoiding artefacts caused by high-frequency, impact-related, oscillations of the measured acceleration pulse. CSDM is proposed by Bandak and Eppinger (1994), which is an indicator of effective "volume fraction" or "occupied volume" of the damaged brain in FE analysis, i.e., the accumulated number of elements in the brain exceeding the specified strain limit, $15 \%$, in stretch was counted, i.e., the score of CSDM was computed based on a maximum principal strain (1st GreenLagrange strain) obtained from the ASCII data output with a 1-ms interval. BTI is a combination of injury assessment parameters involved in translational and rotational acceleration pulses applied to the head CG (Tamura and Yang, 2019). More specifically, BTI was computed on the basis of a pair of representative Injury Assessment Reference Values (IARVs) as given by equation (3). It is worth noting that $\mathrm{HIC}_{15}$ score of 1000 corresponds to a $15 \%$ risk of sustaining an AIS $4+$ (greater than or equal to severe level) TBI (Prasad and Mertz, 1985) and $\ddot{\theta}_{\max } \geq 18 \mathrm{krad} / \mathrm{s}^{2}$ indicates the risk of sustaining a severe diffuse axonal injury (Schmitt et al., 2007), both of which were assigned here as $\mathrm{IARV}_{1}$ and $\mathrm{IARV}_{2}$, respectively. Statistical analysis was performed using IBM SPSS Statistics 24 (IBM Corp., Armonk, NY, USA) with a $P$-value of less than 0.05 considered as statistically significant.

$$
\mathrm{HIC}_{15}=\max \left[\frac{\int_{t_{1}}^{t_{2}} a(t) d t}{t_{2}-t_{1}}\right]^{2.5}\left(t_{2}-t_{1}\right)
$$




$$
\begin{aligned}
& \ddot{\theta}_{\max }=\max \left[\frac{\int_{t_{3}}^{t_{4}} \ddot{\theta}(t) d t}{t_{4}-t_{3}}\right] \\
& \mathrm{BTI}=\frac{\mathrm{HIC}_{15}}{\mathrm{IARV}_{1}}+\frac{\ddot{\theta}_{\max }}{\mathrm{IARV}_{2}}
\end{aligned}
$$

Figure 4 Typical examples of post impact pedestrian kinematics with a soft landing (suv 09 and sdn 02)
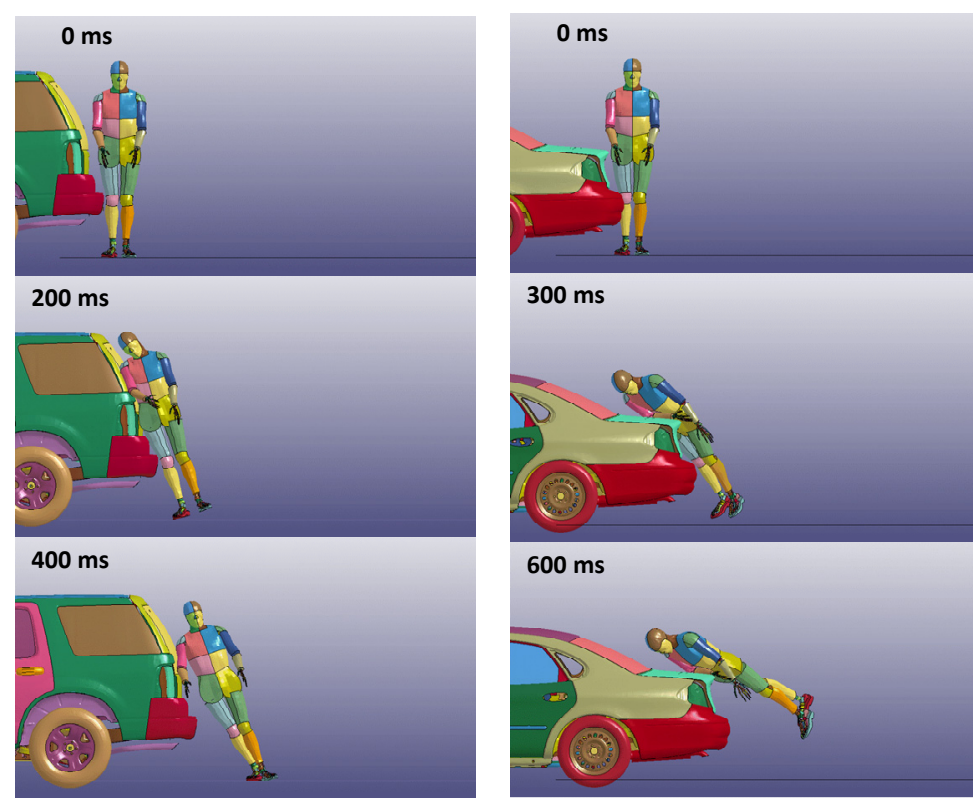

$600 \mathrm{~ms}$

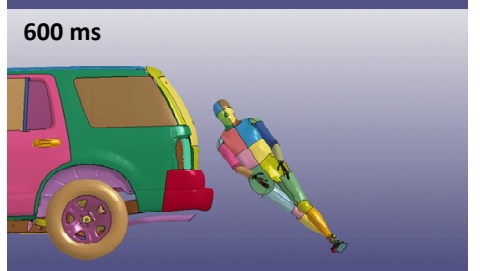

$800 \mathrm{~ms}$

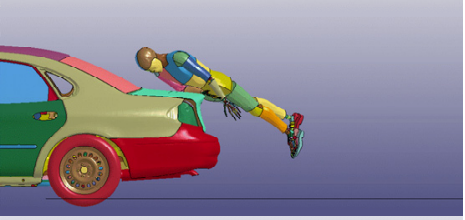

$900 \mathrm{~ms}$
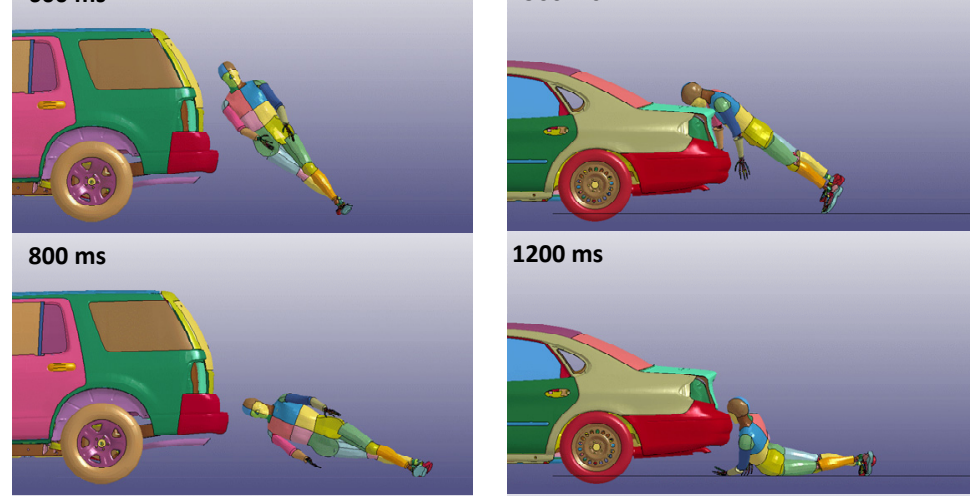

$1200 \mathrm{~ms}$

$1000 \mathrm{~ms}$
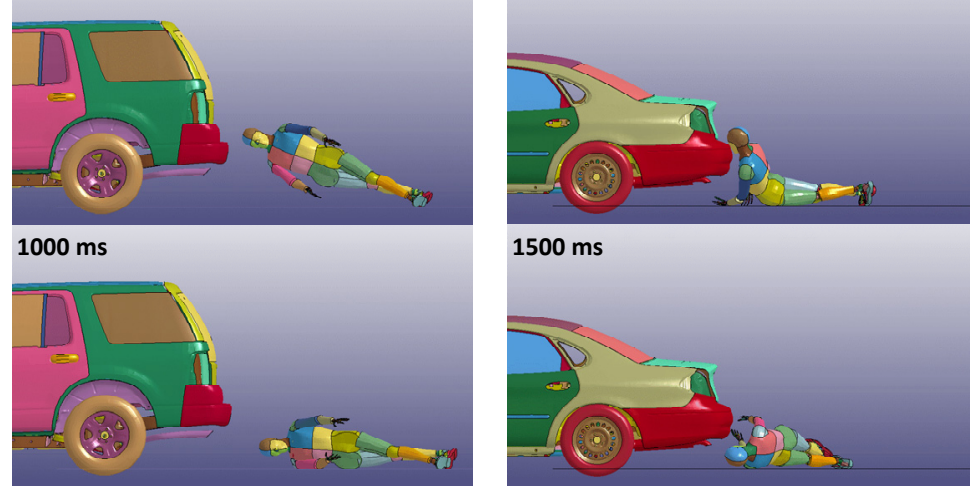

$1500 \mathrm{~ms}$

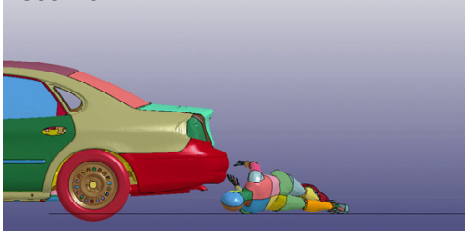




\section{Results}

\subsection{Pedestrian kinematics}

Figures 3 and 4 show typical examples of post impact pedestrian kinematics with a "successful" way of soft landing and the corresponding time history of reaction forces with respect to each body part in the event of a reversing vehicle-to-pedestrian impact through to the final event of ground contact. When struck by the left corner of a reversing SUV (see Figure 4(a)), the standing pedestrian was pushed backward along the direction of the moving vehicle, because the rear window surface of the SUV was relatively flat and had a steep angle. Thus, the struck pedestrian was readily moved along a straight lateral direction and landed on the ground hitting the lower extremity first, followed by the torso and the upper extremity in a sequential order. And finally, the head struck the ground in a relatively "moderate" manner (see Figure 5(a)) since the shoulder effectively shielded the head during the ground impact phase.

On the other hand, when struck by the right corner of a reversing sedan (Figure 4(b)), a walking pedestrian's upper body fell onto the trunk lid and slid down to the ground along the rear surface of the striking car. Since there was an effective mechanical interaction between the pedestrian's upper body and the striking car, a certain amount of impact energy was consumed in comparison with the SUV impact cases. Nevertheless, the head finally impacted against the rear bumper just prior to the ground contact event. Consequently, the struck pedestrian "safely" landed onto the ground without a severe damage to the head (Figure 5(b)). Of note, however, is that CSDM reached approximately $10 \%$ even after the translational and rotational acceleration pulses applied to the head were effectively suppressed under the scores of IARVs, i.e., $\mathrm{HIC}_{15}=1000$ and $\ddot{\theta}_{\max }=18 \mathrm{krad} / \mathrm{s}^{2}$.

\subsection{Injury analysis}

The average $\mathrm{HIC}_{15}$ score of 4191 , obtained in the SUV impact cases, was significantly higher than that involved in the sedan impact cases of $2403\left({ }^{*} P<0.05\right)$, both of which were higher than the recommended IARV score, $\mathrm{HIC}_{15}=1000$ (see Figure 6(a)). We also found a steady increase in $\mathrm{HIC}_{15}$ with the change in pedestrian transverse speed, $V_{y}$; a running pedestrian $\left(V_{y}=4.0 \mathrm{~m} / \mathrm{s}\right)$ was at a significantly greater risk $\left({ }^{*} P<0.05\right)$ of sustaining TBI than a standing pedestrian $\left(V_{y}=0.0 \mathrm{~m} / \mathrm{s}\right)$ (Figure $\left.6(\mathrm{~b})\right)$. There were no significant differences in $\ddot{\theta}_{\max }$ between the striking vehicle types and the pedestrian transverse speeds (see Figure 7). However, the CSDM score was significantly higher for SUV impact cases $\left({ }^{*} P<0.05\right.$ vs. sedan) and running pedestrian cases $\left({ }^{*} P<0.05\right.$ vs. $\left.V_{y}=0.0 \mathrm{~m} / \mathrm{s}\right)($ see Figure 8$)$. Notably, average $\ddot{\theta}_{\max }$ scores were higher than the IARV score of $18 \mathrm{krad} / \mathrm{s}^{2}$ except for a standing pedestrian case $\left(V_{y}=0.0 \mathrm{~m} / \mathrm{s}\right)$. These results indicate that an SUV was more aggressive than a sedan, and a standing pedestrian $\left(V_{y}=0.0 \mathrm{~m} / \mathrm{s}\right)$ was safer than the other impact configurations. 
Figure 5 Typical examples of time history of reaction forces during the reversing vehicle-topedestrian impact with a soft landing. (a) SUV vs. pedestrian (suv 09). (The arrow indicates an event of head contact against the ground) (b) Sedan vs. pedestrian (sdn 02). (The arrow indicates an event of head contact against the rear bumper)

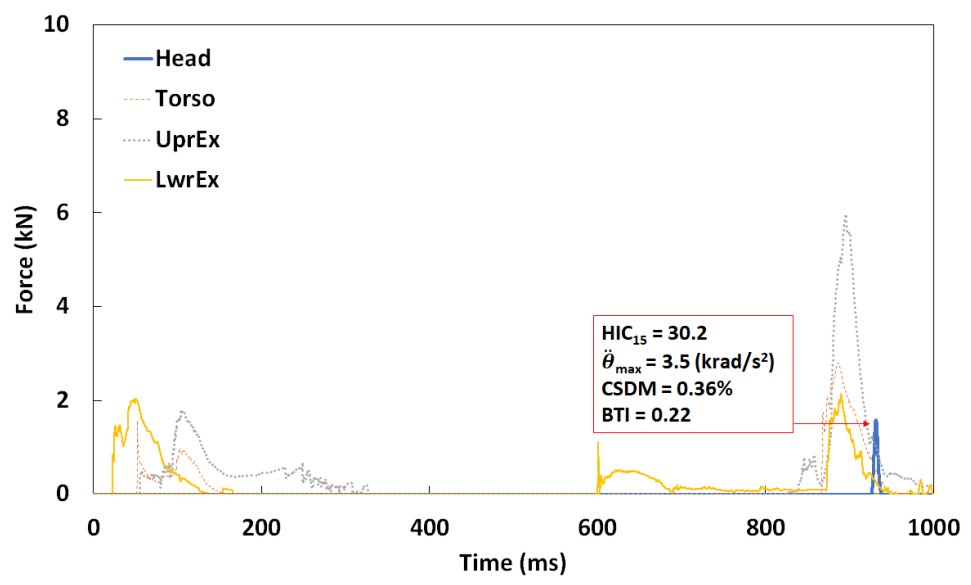

(a)

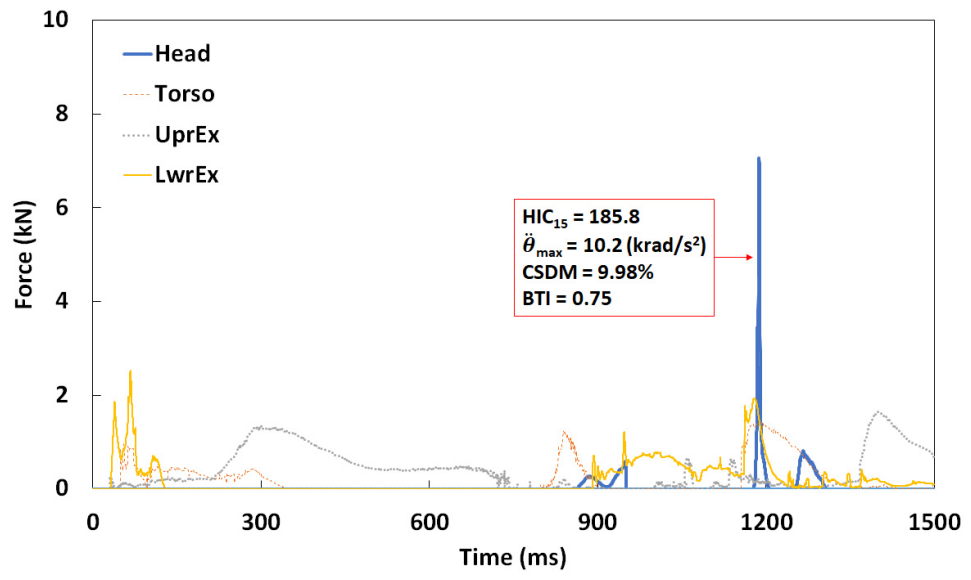

(b) 
Figure 6 Change in Head Injury Criterion, $\mathrm{HIC}_{15}$, involved in ground impact event (mean $\pm \mathrm{SD}$ ). (a) $\mathrm{HIC}_{15}$ represented as a function of striking vehicle type $\left({ }^{*} P<0.05\right.$ for SUV vs. sedan) (b) $\mathrm{HIC}_{15}$ represented as a function of pedestrian transverse speed $\left({ }^{*} P<0.05\right.$ for $V_{\mathrm{y}}=0.0 \mathrm{vs} .4 .0 \mathrm{~m} / \mathrm{s}$ )

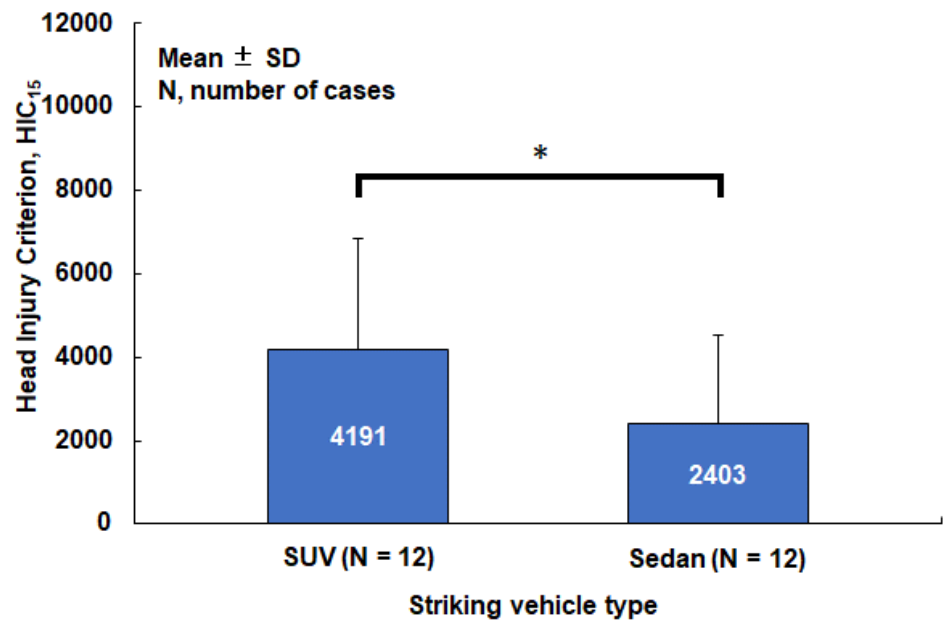

(a)

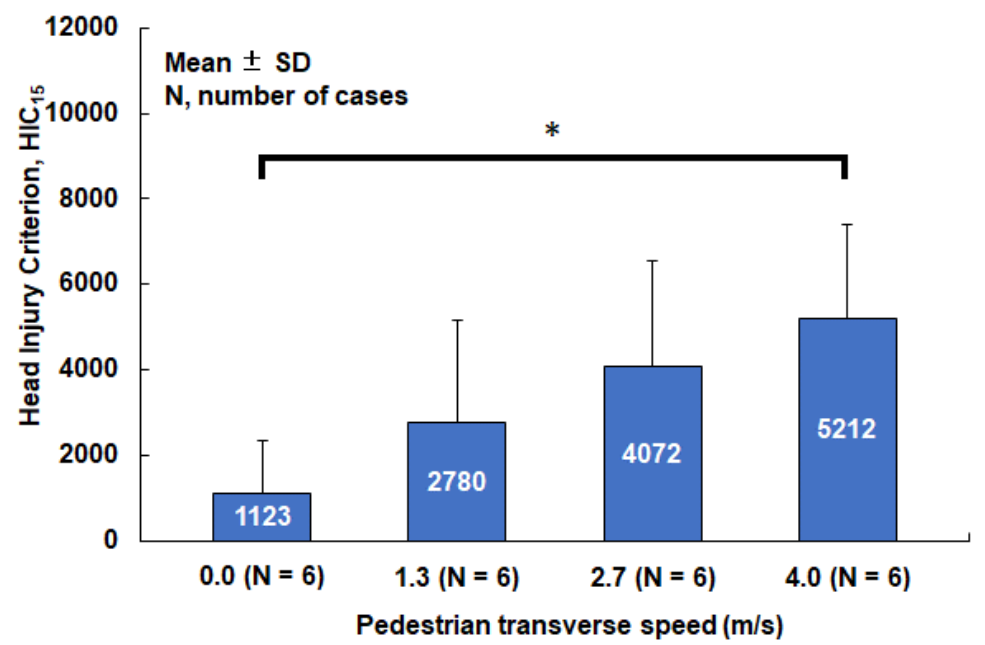

(b) 
Figure 7 Change in maximum head rotational acceleration, $\ddot{\theta}_{\max }$, involved in ground impact event (mean $\pm \mathrm{SD}$ ). (a) $\ddot{\theta}_{\max }$ represented as a function of striking vehicle type (b) $\ddot{\theta}_{\max }$ represented as a function of pedestrian transverse speed

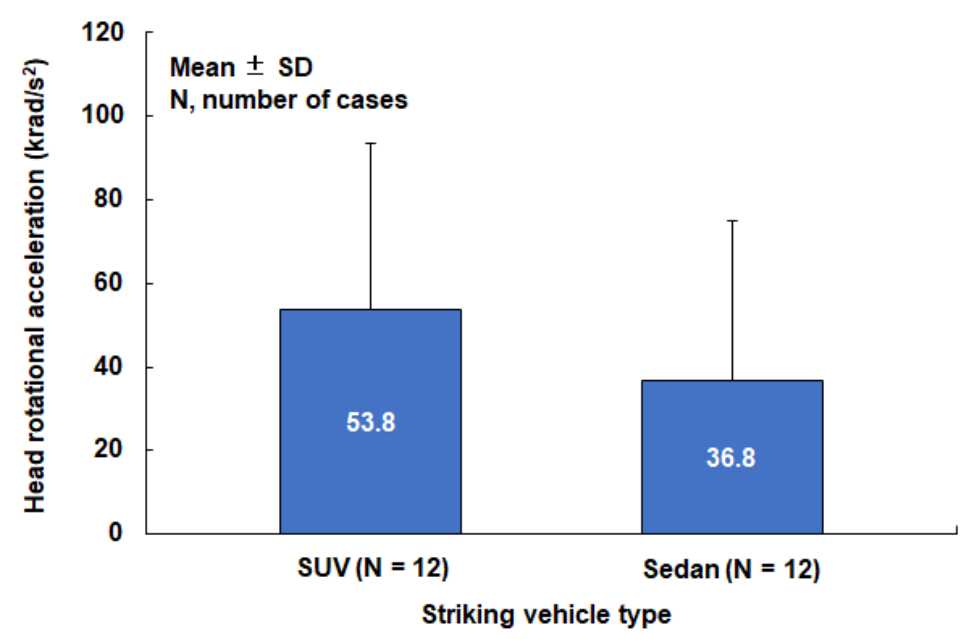

(a)

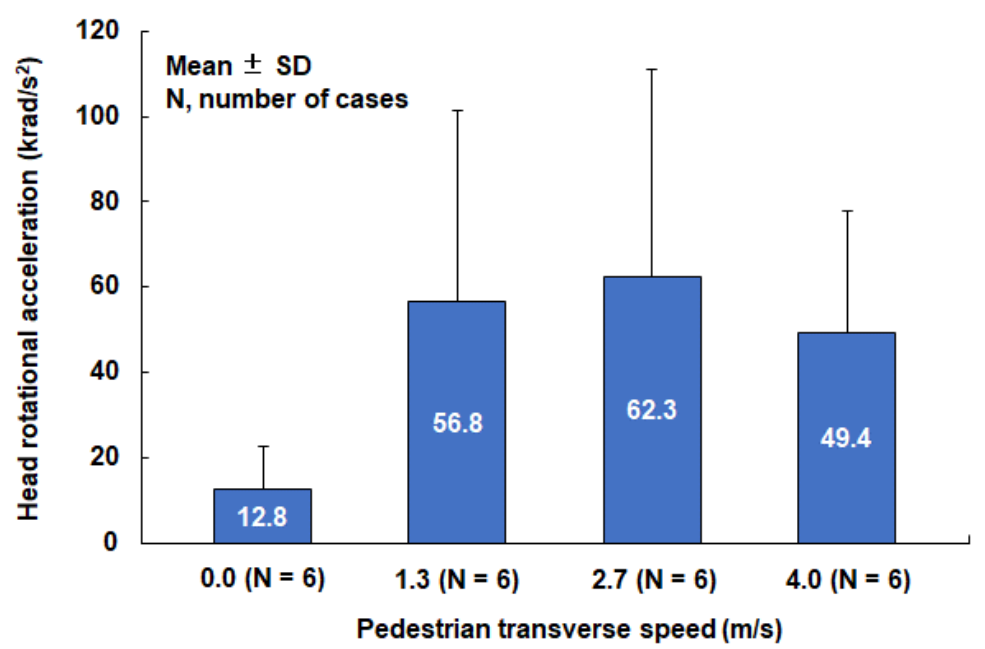

(b) 
Figure 8 Change in cumulative strain damage measure, CSDM, involved in ground impact event (mean $\pm \mathrm{SD}$ ). (a) CSDM represented as a function of striking vehicle type $\left({ }^{*} P<0.05\right.$ for SUV vs. sedan) (b) CSDM represented as a function of pedestrian transverse speed $\left({ }^{*} P<0.05\right.$ for $V_{\mathrm{y}}=0.0$ vs. $\left.4.0 \mathrm{~m} / \mathrm{s}\right)$

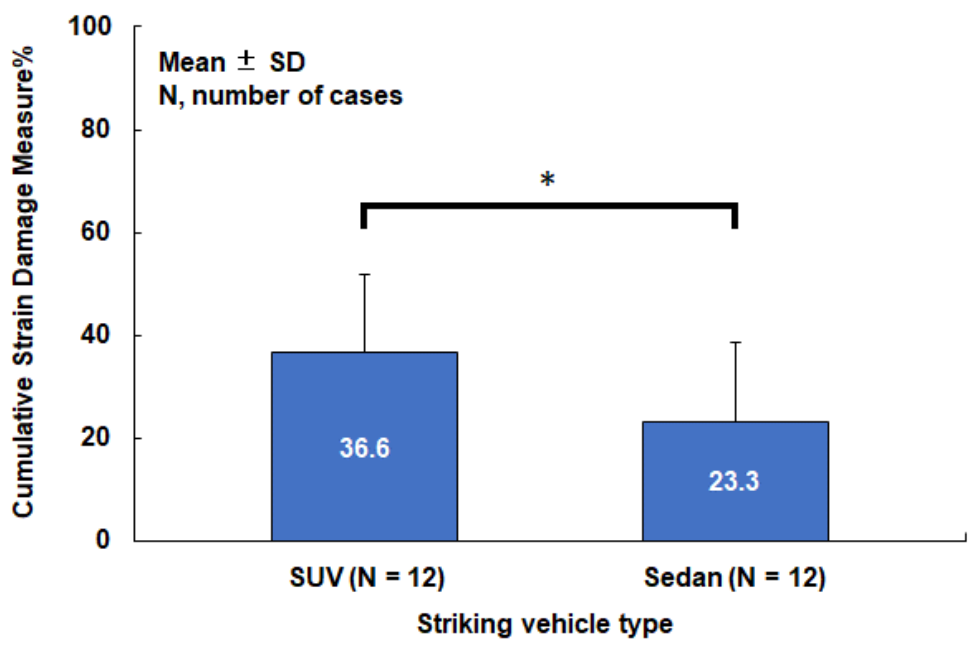

(a)

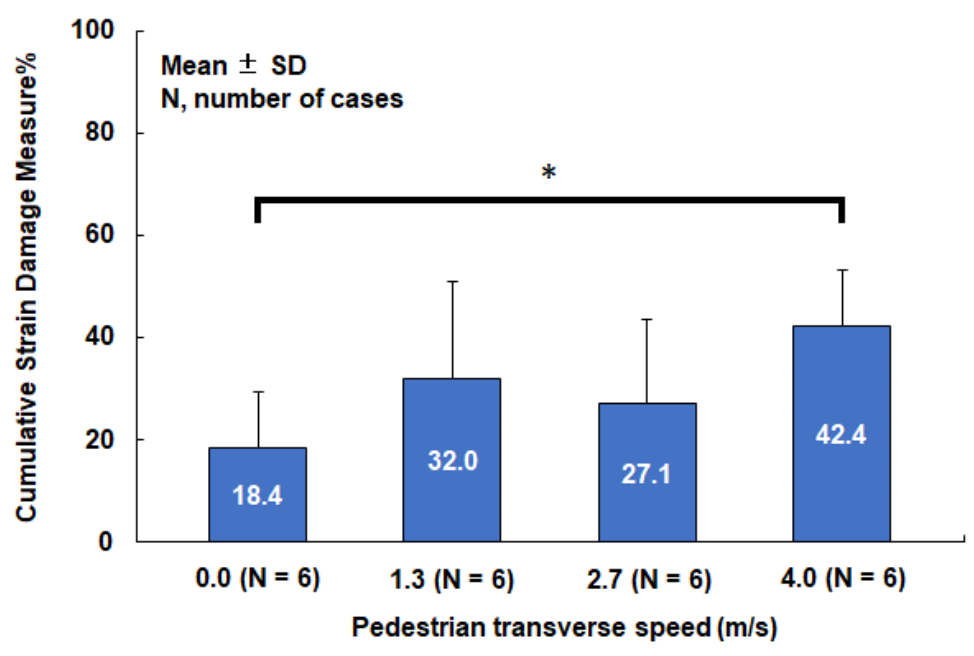

(b)

We observed a similar trend in the BTI (see Figure 9), wherein the SUV was more aggressive than the sedan, and jogging or running pedestrians $\left(V_{\mathrm{y}}=2.7\right.$ or $\left.4.0 \mathrm{~m} / \mathrm{s}\right)$ were significantly more susceptible to sustaining TBI in comparison with a standing pedestrian $\left(V_{\mathrm{y}}=0.0 \mathrm{~m} / \mathrm{s}\right)\left({ }^{*} P<0.05\right)$. However, the impact location (right, left and centre) of the struck pedestrian did not significantly affect $\mathrm{HIC}_{15}, \ddot{\theta}_{\max }$, CSDM and BTI scores. 
Figure 9 Change in brain trauma index, BTI, involved in ground impact event (mean \pm SD). (a) BTI represented as a function of striking vehicle type (b) BTI represented as a function of pedestrian transverse speed $\left({ }^{*} P<0.05\right.$ for $V_{\mathrm{y}}=0.0 \mathrm{vs} .2 .7$ and $\left.4.0 \mathrm{~m} / \mathrm{s}\right)$

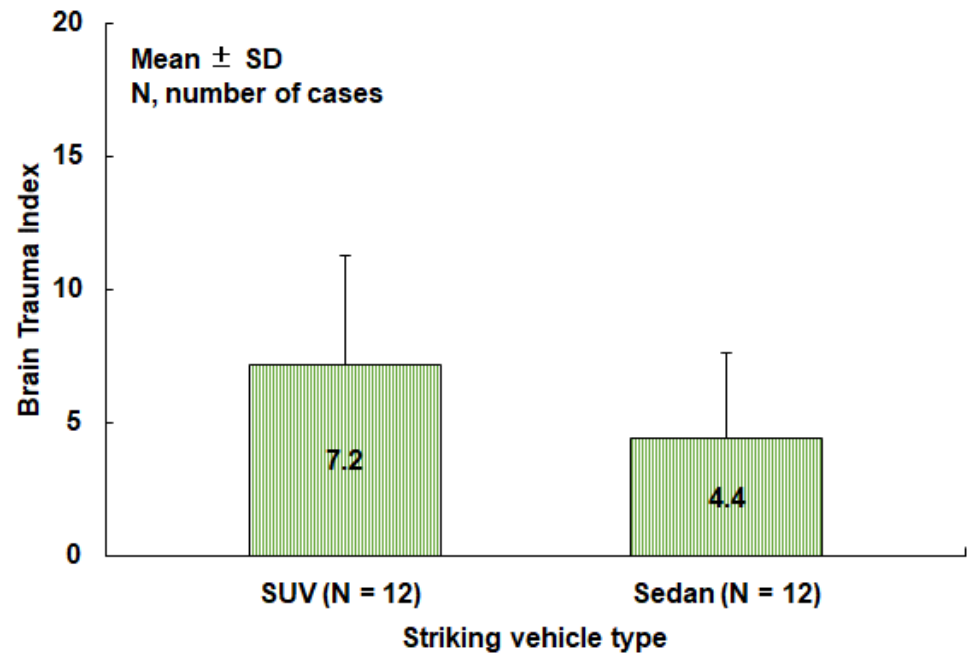

(a)

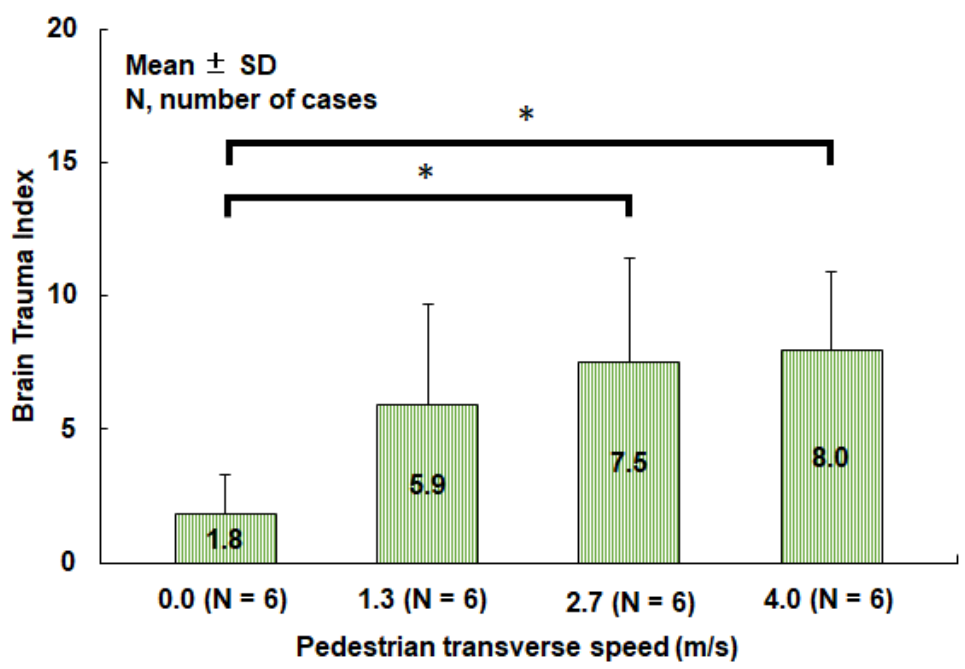

(b)

Comparison of throw distances of a struck pedestrian in $X$ and $Y$ directions showed that pedestrians impacted by an SUV were thrown further away, $1247 \mathrm{~mm}$ on average, in the direction of the moving vehicle than those impacted by a sedan, $390 \mathrm{~mm}\left({ }^{* *} P<0.01 \mathrm{vs}\right.$. sedan) (see Figure 10). Further, jogging or running pedestrians were thrown further away, $-2043 \mathrm{~mm}$ on average, in the perpendicular direction to the moving vehicle than standing or walking pedestrians, $-403 \mathrm{~mm}\left({ }^{* * *} P<0.001\right.$ vs. low $\left.V_{\mathrm{y}}\right)$. These results are consistent with the representative post impact pedestrian kinematics shown in Figure 4. 
Figure 10 Throw distances were calculated on the basis of the head CG moving distance of a struck pedestrian (mean $\pm \mathrm{SD}$ ). Note that low $V_{\mathrm{y}}=0.0$ and $1.3(\mathrm{~m} / \mathrm{s})$ and high $V_{\mathrm{y}}=2.7$ and $4.0(\mathrm{~m} / \mathrm{s})$, respectively. (a) Throw distance represented as a function of striking vehicle type $\left({ }^{* *} P<0.01\right.$ for SUV vs. sedan in $\left.X\right)$ (b) Throw distance represented as a function of pedestrian transverse speed $\left({ }^{* * *} P<0.001\right.$ for low $V_{\mathrm{y}}$ vs. high $V_{\mathrm{y}}$ in $Y$ )

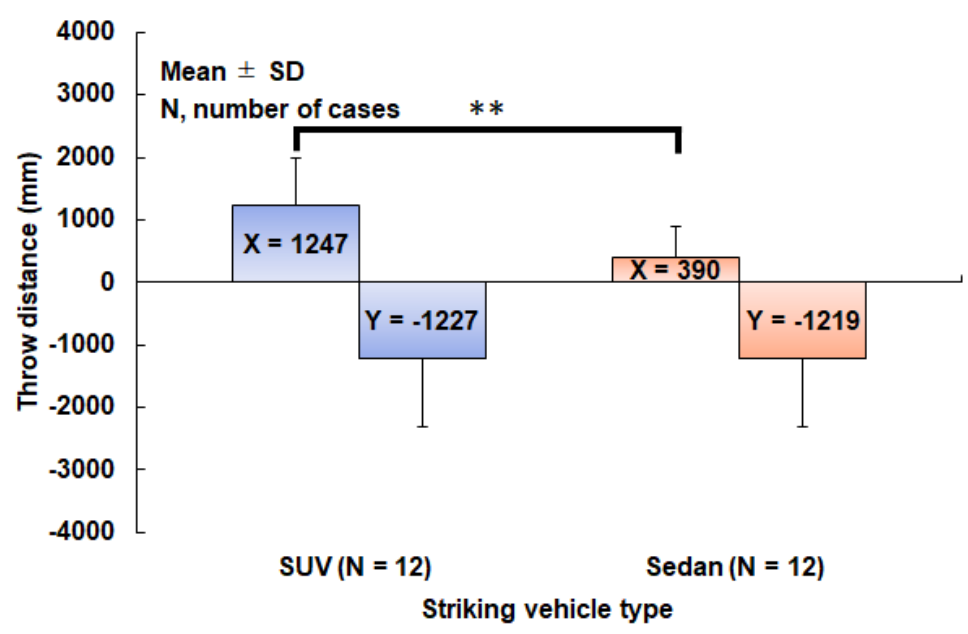

(a)

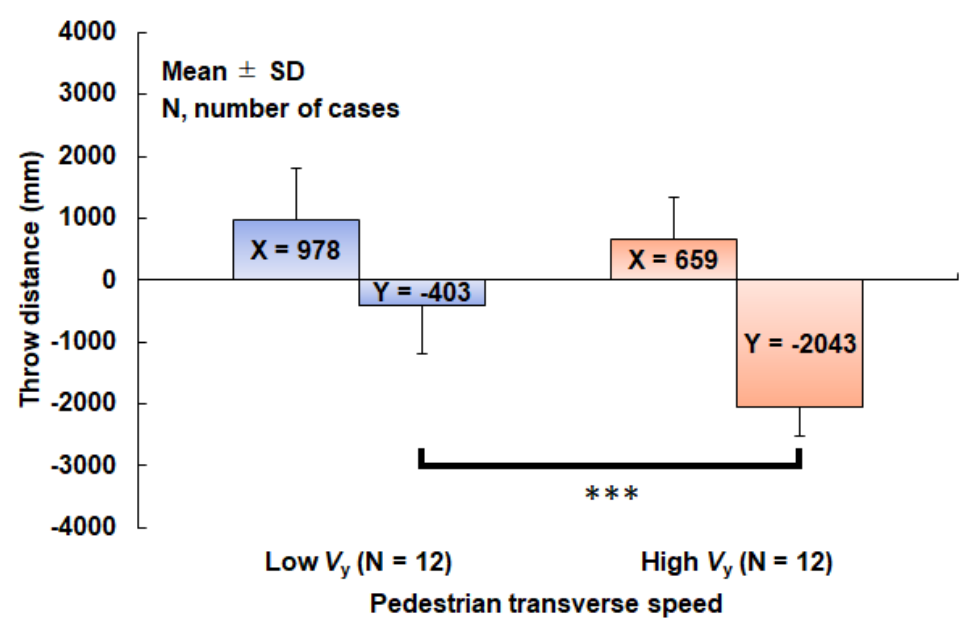

(b)

\section{Discussion}

Backover associated injuries sustained in a reversing vehicle-to-pedestrian impact are a significant off-road safety concern. However, their prevalence is relatively underestimated at present as the majority of such injuries fall outside the scope of official road injury recording systems or accident databases. Thus, backover injury is not mostly 
recorded in police-reported data, which focus on incidents and accidents that occur on public roads (Keall et al., 2017). Recently, Lindman et al. (2011) analysed a total of 330, real-world, car-to-pedestrian accidents including frontal and rear collisions and found that backover crashes are not necessarily rare $(25 \%)$ compared with frontal collisions (75\%); the most frequent AIS 2+ injured body parts in backover crashes were the head $(27 \%)$ followed by the lower extremity $(25 \%)$ and the upper extremity $(20 \%)$. Of note, they also reported that the most frequent contact area was the rear bumper. However, it remains unsure whether or not the injuries to the head were primarily caused by contact against the ground or the striking car. Further, the factor contributing most to backover collisions was "failed to look" for drivers and pedestrians (Fildes et al., 2017) or that either the driver or the pedestrian did not look properly during a reversing manoeuvre. In the present study, therefore, we assumed that the representative backover crash configurations can be described such that a standing or moving pedestrian was struck laterally by a low-speed reversing vehicle.

Post impact pedestrian kinematics in this study were completely different depending on the striking vehicle types. In an SUV-to-pedestrian impact, the rear surface of the striking vehicle was relatively flat with a steep angle, and struck pedestrians were readily thrown further away in the direction of the moving vehicle. In contrast, a sedan-topedestrian impact showed the struck pedestrian falling onto the trunk lid, and it is likely that a major part of the impact energy was effectively consumed as kinetic energy prior to the ground impact phase. Hence, each of the injury assessment scores related to translational and rotational acceleration pulses applied to the head was remarkably higher for the SUV than the sedan simulated in this study. Both translational and rotational accelerations significantly exceeded the representative scores of IARVs, i.e., HIC $\mathrm{H}_{15}=$ 1000 and $\ddot{\theta}_{\max }=18 \mathrm{krad} / \mathrm{s}^{2}$. We also found that pedestrian transverse speed significantly contributed to the severity of $\mathrm{TBI}$ risk for $\mathrm{HIC}_{15}$ and $\mathrm{CSDM}$; i.e., a running pedestrian was more susceptible to sustaining severe TBI compared with a standing pedestrian even in a low-speed backover collision. The locations of the striking vehicle that impacted the struck pedestrian were not significantly influential. Nevertheless, in the current impact configurations, a pedestrian struck by the left corner with high $V_{\mathrm{y}}(2.7$ or $4.0 \mathrm{~m} / \mathrm{s})$ would be at a greater risk, because a mechanical interaction between the vehicle structure and the pedestrian body was extremely limited, whereas the whole kinetic energy ended up being consumed by ground impact phase in these cases. Since the impact velocities were generally lower in reversing accidents, it is likely that pedestrian transverse speed effectively contributed to the increase in resultant impact velocity, leading to high throw distance in the direction perpendicular to that of the moving vehicle. Thus, a running pedestrian $\left(V_{\mathrm{y}}=4.0 \mathrm{~m} / \mathrm{s}\right)$ struck by a reversing vehicle at $10 \mathrm{~km} / \mathrm{h}$ produces an impact velocity equivalent to $17.5 \mathrm{~km} / \mathrm{h}$, which is unexpectedly higher than the initially designated reversing vehicle velocity.

In this work, BTI is computed on the basis of translational and rotational acceleration pulses applied to the head. Similar to other injury assessment parameters, BTI also clearly indicated that an SUV was more aggressive than a sedan when ground impact phase was considered and that swiftly moving pedestrians were more susceptible to sustaining TBI. Despite the lack of a close relationship between BTI and other mechanical parameters due in part to the limited number of cases simulated here $(N=24)$, the change in BTI scores was generally consistent with the corresponding results obtained for $\mathrm{HIC}_{15}, \ddot{\theta}_{\max }$ and CSDM. 
This study is not without limitations, including the effects of age and its concomitant inherent properties. The lack of active muscle tone effect was also a limitation in this study. TBI risk could be alleviated by replicating natural avoidance behaviour and protective posturing prior to the impending collision or final impact with the ground. However, previous studies demonstrated that elderly people are exposed to greater risk of serious injuries (Fildes et al., 2017; Keall et al., 2017), which may be attributed to reduced bone strength, poor balance and slower reaction time, etc., and human active reaction will not greatly influence findings obtained in the current study. In fact, Alvalez et al. (2013 and 2014) performed a series of numerical simulations by varying the vehicle speed, pedestrian initial posture and muscle tone effect and investigated the influence of a neck muscle tones on head kinematics during pedestrian accidents. It was concluded that for the muscle tones used in their study, the influence on the resultant strain in the brain tissue was minor, while the head impact location and direction were influenced by the tensed neck musculature. Thus, implementing an automatic emergency braking with backing assistance systems such as rear-view cameras and rear parking sensors will potentially mitigate the severity of TBI involved in low-speed backover crashes (Fildes et al., 2017; Keall et al., 2017; Cicchino, 2019a, 2019b).

In addition to such muscle tone effects on the severity of adult pedestrian injuries, small children are vulnerable to this type of backover accident. According to a retrospective review study conducted in the USA (Fenton et al., 2005), it was found that trucks and SUVs with larger blind spots were more often involved in accidents affecting children aged 5 years or younger. The most common injuries were abrasions $(52 \%)$ and blunt head injuries $(48 \%)$ with a mortality rate of $8.2 \%$. Since young children with a short stature were more commonly involved in private driveway-related vehicle-topedestrian accidents, they sustain TBI differently compared with adults and older children who are more likely to be injured by falling from a moving vehicle. Thus, the mechanism of TBI for younger children remains under-recognised at this moment and should be elucidated earnestly. In the current series of reversing impact cases, we confirmed that a soft landing was attainable and was effective at protecting the head during the final event of ground contact. Nevertheless, since bumper-to-head contact can be injurious even in a low-speed backover crash involving sedans, it may be recommended that the rear surface configuration of vehicles be improved in the future.

In conclusion, to the authors' knowledge, this is the first study to reveal that the risk of sustaining a severe TBI is likely high, even in low-speed backover collisions. This event is probably underreported, and its injury severity may have been overlooked and underestimated so far. By conducting a series of reversing vehicle-to-pedestrian impact simulations, however, we found that backover injuries are particularly injurious when the ground impact event is considered.

\section{Acknowledgements}

The authors are deeply grateful to Dr. King, a Distinguished Professor at Wayne State University, Detroit, Michigan (MI), USA, with whom a private discussion originally motivated a series of our ground-related pedestrian impact studies. This work was partially supported by a KAKENHI Grant-in-Aid (No. 19K12782) for Scientific Research (C). 


\section{References}

Alvarez, V., Fahlstedt, M., Halldin, P. and Kleiven, S. (2013) 'Importance of neck muscle tonus in head kinematics during pedestrian accidents,' Proceedings of the International Research Council on Biomechanics of Injury (IRCOBI), Paper No. IRC-13-91, Gothenburg, Sweden.

Alvarez, V.S., Halldin, P. and Kleiven, S. (2014) 'The influence of neck muscle tonus and posture on brain tissue strain in pedestrian head impacts,' Stapp Car Crash Journal, Vol. 58, pp.63-101.

Austin, R. (2008) Fatalities and Injuries in Motor Vehicle Backing Crashes, Report to Congress, National Highway Traffic Safety Administration, U.S. Department of Transportation, Washington, DC. Available online at: http:/www-nrd.nhtsa.dot.gov/Pubs/811144.pdf (accessed on 5 May 2019).

Bandak, F. and Eppinger, R. (1994) 'A three-dimensional finite element analysis of the human brain under combined rotational and translational accelerations', Proceedings of the 38th Stapp Car Crash Conference, Paper No. 942215, Fort Lauderdale, FL, USA.

Cicchino, J.B. (2019a) 'Real-world effects of rear automatic braking and other backing assistance systems,' Journal of Safety Research, Vol. 68, pp.41-47.

Cicchino, J.B. (2019b) 'Real-world effects of rear cross-traffic alert on police-reported backing crashes,' Accident Analysis and Prevention, Vol. 123, pp.350-355.

Crocetta, G., Piantini, S., Pierini, M. and Simms, C. (2015) 'The influence of vehicle front-end design on pedestrian ground impact,' Accident Analysis and Prevention, Vol. 79, pp.56-69.

Decker, S., Otte, D., Cruz, D.L., Müller, C.W., Omar, M., Krettek, C. and Brand, S. (2016) 'Injury severity of pedestrians, bicyclists and motorcyclists resulting from crashes with reversing cars,' Accident Analysis and Prevention, Vol. 94, pp.46-51.

Fenton, S.J., Scaife, E.R., Meyers, R.L., Hansen, K.W. and Firth, S.D. (2005) 'The prevalence of driveway back-over injuries in the era of sports utility vehicles,' Journal of Pediatric Surgery, Vol. 40, pp.1964-1968.

Fildes, B., Keall, M.D. and Newstead, W. (2017) 'Backover collision and the effectiveness of reversing cameras', Proceedings of the 25th International Technical Conference on the Enhanced Safety of Vehicles (ESV), Paper No. 17-0250, Detroit, MI, USA.

Keall, M.D., Fildes, B. and Newstead, S. (2017) 'Real-world evaluation of the effectiveness of reversing camera and parking sensor technologies in preventing backover pedestrian injuries,' Accident Analysis and Prevention, Vol. 99, pp.39-43.

Lindman, M., Jakobsson, L. and Jonsson, S. (2011) 'Pedestrians interacting with a passenger car; a study of real world accidents', Proceedings of the International Research Council on the Biomechanics of Injury (IRCOBI), Paper No. IRC-11-61, Krakow, Poland.

NHTSA (2010) Federal Motor Vehicle Safety Standard, Rearview Mirrors; Federal Motor Vehicle Safety Standard, Low-Speed Vehicles Phase-in Reporting Requirements, Docket No. NHTSA2010-0162. Available online at: http://www.nhtsa.gov/staticfiles/rulemaking/pdf/ Rear_Visibility_NPRM_12032010.pdf(accessed on 5 May 2019).

NHTSA Crash Simulation Vehicle Models Available online at: https://www.nhtsa.gov/crashsimulation-vehicle-models (accessed on 5 May 2019).

Prasad, P. and Mertz, H. (1985) 'The position of the United States delegation to the ISO Working Group 6 on the use of HIC in the automotive environment,' Proceedings of SAE Government Industry Meeting and Exposition, Paper No. 851246, Washington DC, USA.

Schmitt, K-U., Niederer, P.F., Muser, M.H. and Walz, F. (2007) Trauma Biomechanics: Accidental Injury in Traffic and Sports, 2nd ed., Springer-Verlag, Berlin, Heidelberg.

Simms, C.K. and Wood, D.P. (2006) 'Effects of pre-impact pedestrian position and motion on kinematics and injuries from vehicle and ground contact,' International Journal of Crashworthiness, Vol. 11, pp.345-355. 
Simms, C.K., Wood, D.P. and Walsh, D.G. (2004) 'Confidence limits for impact speed estimation from pedestrian projection distance,' International Journal of Crashworthiness, Vol. 9, pp.219-228.

Tamura, A. and Yang, K.H. (2019) 'Essential factors leading to a traumatic brain injury during low-speed fender vault pedestrian impacts,' International Journal of Vehicle Safety, Vol. 11, pp.1-18.

Tamura, A., Hasegawa, J. and Koide, T. (2018) 'Comparative analysis on traumatic brain injury risk due to primary and secondary impacts in a pedestrian sideswipe accident,' ASCE-ASME Journal of Risk and Uncertainty in Engineering Systems, Part B: Mechanical Engineering, Vol. 4, pp.041004-4-041004-7.

Tamura, A., Koide, T. and Yang, K.H. (2016) 'Effects of translational and rotational accelerations on traumatic brain injury in a sport utility vehicle-to-pedestrian crash,' International Journal of Vehicle Design, Vol. 72, pp.208-229.

Wood, D.P., Simms, C.K. and Walsh, D.G. (2005) 'Vehicle-pedestrian collisions: validated models for pedestrian impact and projection,' Proceedings of the Institution of Mechanical Engineers: Journal of Automobile Engineering, Vol. 219, pp.183-195. 